\title{
The study and optimization of the behavior and energy absorbed in the frontal impact by a rectangular metal workpiece with an Origami core
}

\author{
Robert-Marian Bleotu, ${ }^{1, *}$ \\ 1'Lucian Blaga" University of Sibiu, Faculty of Engineering, Str. Emil Cioran Nr. 4, 550025, \\ Romania
}

\begin{abstract}
The bumper systems (beams and face bars) are parts of the car body structure, one of the most important components of an auto vehicle because of its role in absorbing the energy of an impact by deformation. The main objective of this paper is to study, optimize the built shape of the frontal members beams used in the endurance structure of motor vehicles in terms of their ability to absorb internal energy resulting from a frontal impact under the principles of sustainability. The study combines the classical technology used in the construction of vehicles with ,the Origami Engineering" technique, which is generally used by NASA, but also by engineers in other fields: aeronautics, nanotechnology or medical technique. Simulation analyses were performed using the finite element on different types of thin-walled metal tubes, but also an origami structure.
\end{abstract}

\section{Introduction}

A generally known definition for sustainable development shows that this is the "development that meets the needs of the present without compromising the ability of future generations to meet their own needs". The embodiment of all the results of the human interaction with the environment was named carbon footprint: "Carbon footprint is one way to illustrate humans' impact on the environment. It is quantified by converting our use of fossil fuels (e.g. for electricity, heating and transportation) to metric tons of carbon dioxide emissions" [1]. The ability of the environment to meet the needs of the present and the future is depicted also in [2]. To reduce the carbon footprint, it is necessary to take into account the principles of sustainability. In the last decades, sustainability has become a presence and a necessity of our existence. Thus, it is the subject of interest for numerous research groups. As an example, the automotive industry, the largest private investor, needs to be mentioned. Source principal reflector of the sustainability principles embodied in automotive manufacturing are: the lightweight design, a sustainable manufacturing processes, a sustainable technology, etc. The lightweight design can be realized through different ways based on new lighter materials or based on the optimization of the system's

${ }^{*}$ Corresponding author: robert.bleotu@ulbsibiu.ro 
constructive form. In this way, the functional role is still accomplished but with less material consumed by the use of technologies and processes. The social, economic and environmental aspects are necessary to be taken into account for a sustainable processing. A large percentage of the processing total cost is represented by the energy consumption of processing. Energy consumption actually reaches the finished product, which is a critical aspect in the analysis of sustainability.

The paper aims to use origami techniques to optimize the constructive shape of the side member. The name „Origami” comes from the Japanese words „ori” which means to fold up and „kami”- which means paper. As the name suggests, origami is the ancient art of folding paper, the purpose being to create figures by geometric folding, without cutting the paper [3].

The "Origami Engineering" technique intersects the art and the engineering in a new concept that greatly helps researchers to obtain complex shapes with very good capabilities such as: compaction, reduction of total mass or absorption of internal energy. These concepts made with the help of the Origami Engineering technique are widely used by NASA researchers for space technology. They are also used in fields such as: underwater research, medical nanotechnology, aerospace, defense, medical or vehicle construction technologies [4].

Numerous studies have been performed to optimize the constructive shape of the side member, given its importance in the car structure. In the context of manufacturing based on sustainable technologies, the main research directions are those related the mass reduction, taking into account the increasing or maintaining a constant level of absorption by:

a) Carrying out tests to highlight the bending behavior of thin-walled tubs [5], tests of the energy absorption mechanism when required for the compression (with the necessary testing facilities) [6-8], tests aimed to study the deformation of thinwalled tubes when they are subject to compression, but also tests for the maximum force that can be supported by them [9];

b) Studies on attempt to mix the mass and increase the energy absorption capacity by using various materials in the construction of these structures: brass, stainless steel [10], magnesium alloys [11], and carbon fiber reinforced tubes [12];

c) Research aimed at optimizing the constructive shape by increasing the absorption of a large amount of energy through various configurations that have as basic elements certain cuts on the surface of the tube: rectangular or special shapes windows [13-15];

d) Studies on the deformation behavior in the case of different tube sections: elliptical with variable section [16], cylindrical [17], conical [18], or hexagonal $[19,20]$;

e) Study of the use of origami tubes in engineering: Sandwich panels are extremely used in the energy industry. They have very good energy absorption and total mass reduction properties. These tubes are often used in the aerospace industry, the construction equipment industry and in other areas where passenger safety is essential. For this purpose, the Miura-ori technique can be used from the simple paper folding technique to fields of modern engineering. The aim is to apply this technique to create a structure that is able to absorb as much energy as possible when subjected to compression stresses. Road accidents are real cases where the compression stresses are found and they have attracted the attention of all car designers. That is why the paper aims to investigate the best solution in energy absorption in case of a car accident. All the tests were performed on boxes and tubes realized by the specialists in the "Origami engineering" technique. 


\section{Case study}

\subsection{Methodology}

The side members are metal bodies, usually made of aluminum or steel. They are located in the front of the vehicles and are mounted by removable or non-removable assembly following the chassis. Their role is to ensure the highest energy absorption in the event of frontal impact, but also to support the assembling of certain auxiliary components of the vehicle [21]. The main strength structure components are depicted in Figure 1.

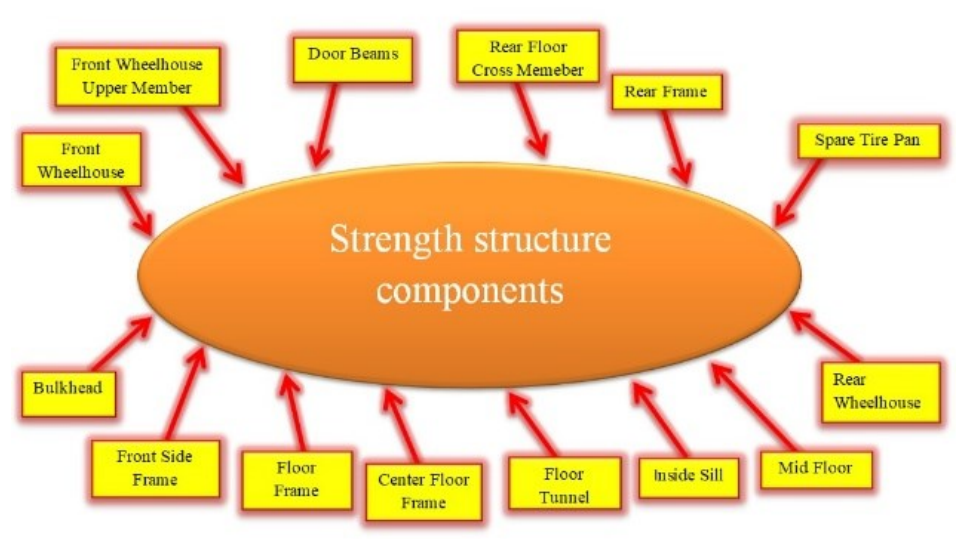

Fig. 1. Strength structure components.

Their shape and model differ from car to car depending on the importance placed by the manufacturer on this very important part of the car.

The actual research studies are aimed at optimizing the form in which the principles of sustainability are taken into account. The most important characteristic of these components, namely the absorption of internal energy in case of a frontal impact must be also highlighted in these studies. To achieve these two characteristics, the Origami Engineering technique will be used.

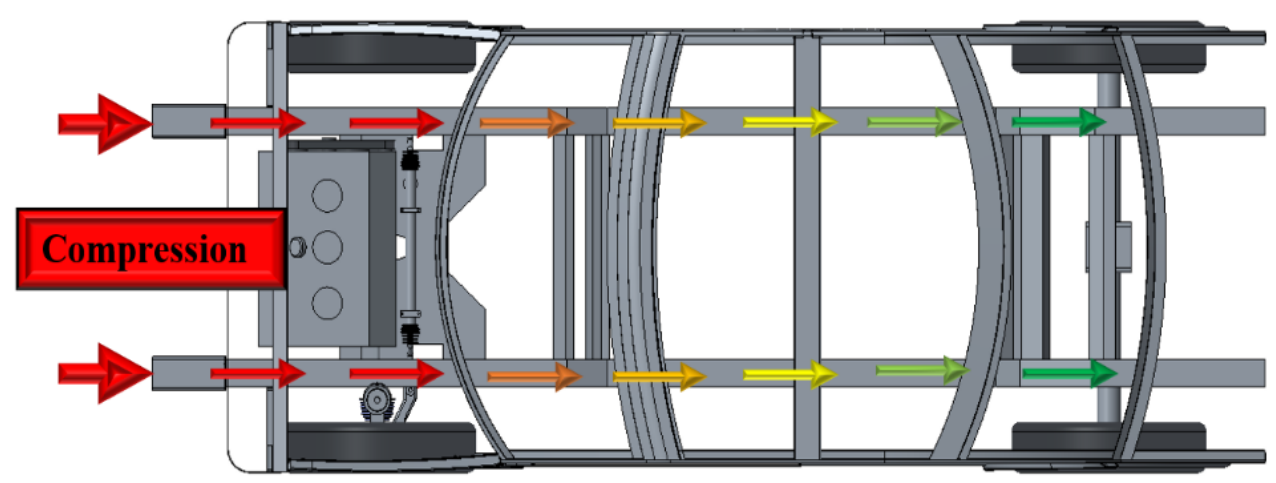

Fig. 2. The side members position and stress. 


\subsection{Representative form - Side members}

The side members are found in different constructive forms, from simple to high complexity forms. Most of these side members are made of extruded tubes that have ribs and additional elements to stiffen their structures, but this stiffening increases its mass and production cost. Such a side member is shown in Figure 3. This model was made using the Creo 3.0 program. The component elements of this side member are: the extruded tube, the sole, inner fittings, exterior reinforcement, headlight support, stiffening rod and fasteners. The tube and the sole of the side member are made of aluminum, while the other components are made of steel.

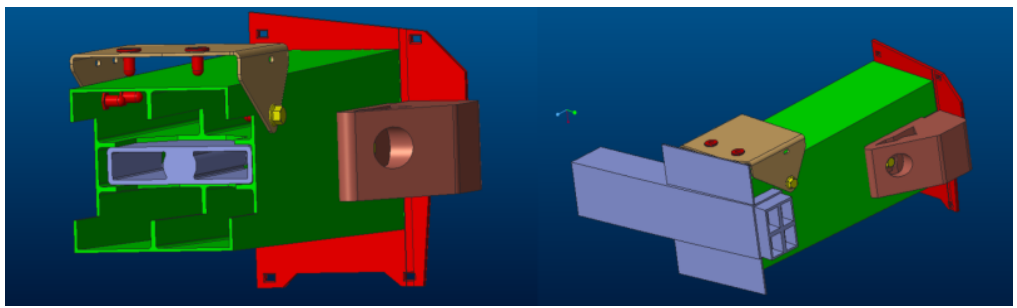

Fig. 3. Side member.

\subsection{Simulation}

In order to fulfill the principles of sustainability, an attempt will be made to reduce the total mass of the side member. This will be achieved by replacing its additional bodies that have the role of stiffening the side member with lighter structures made by Origami Engineering technique. Table 1 shows the elements that are replaced with the new concept consisting of tube and origami structure.

Table 1. Application of the principles of sustainability.

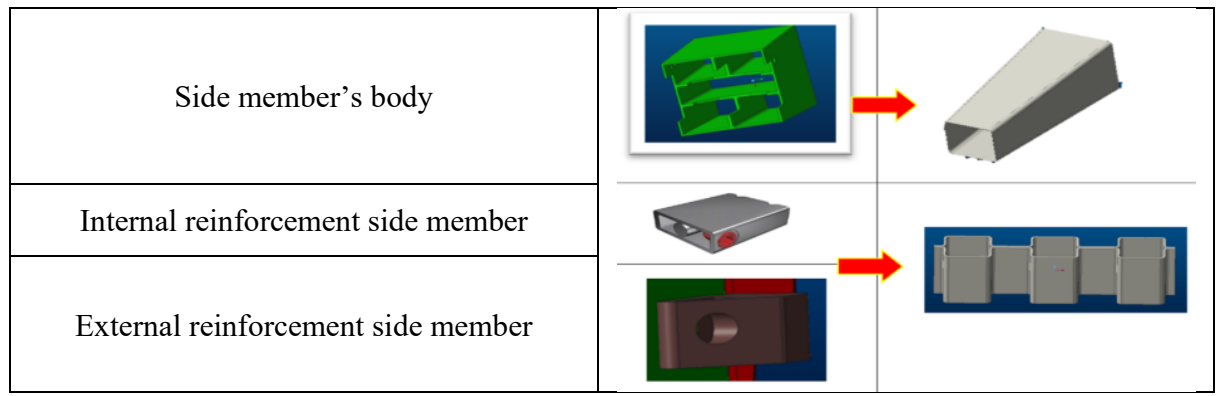

The finite element simulation of the wall tubes was performed with the help of the Abaqus program. This program helps to simulate solid bodies or those made of surfaces, in terms of their ability to deform or absorb a certain amount of energy, if they are subjected to stress.

\subsubsection{Cross section of the tube}

Four types of tubes were used to perform this analysis, with a length of $300 \mathrm{~mm}$ and a total width of $100 \mathrm{~mm}$. What differs in these four tubes is the cross section, so the following sections are identified: circle, hexagon, square and triangle. Their modeling was done in the 
surface model of the CREO program, to then be given a thickness of $2 \mathrm{~mm}$ directly from the Abaqus simulation program.

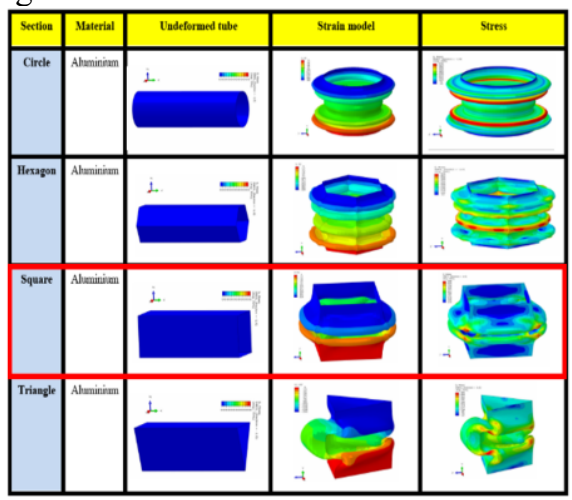

Fig. 4. Simulation obtained for the cross section of the tube.

Following the analysis performed on tubes with different transverse shapes, it can be seen that the square section obtained the best result in terms of energy absorption, namely $39,000 \mathrm{~mJ}$ compared to the triangle which obtained only $17,000 \mathrm{~mJ}$, the analysis of this tube stopping at a displacement of $150 \mathrm{~mm}$. The other two tubes obtained results close to the square section tube.

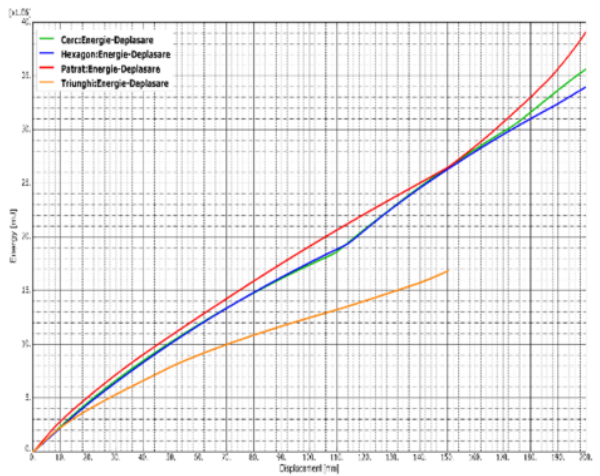

Fig. 5. Simulation graph by cross section of the tube.

The maximum stress at the thin-walled tube that has a square cross section, and that obtained the best results in terms of absorption is $684 \mathrm{MPa}$. These appear as can be seen at the corners of the tube, which shows us rays. Its deformation on the $\mathrm{Y}$ axis reaches a value of $200 \mathrm{~mm}$ at the end of the axial compression stress.

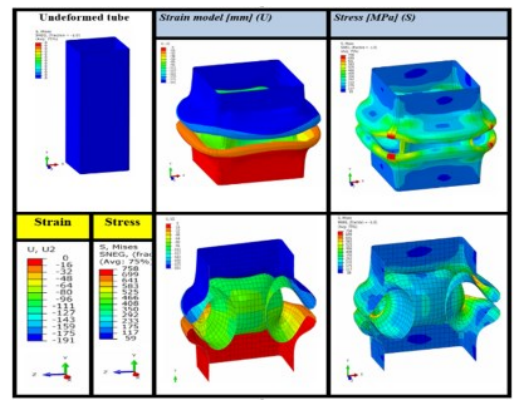

Fig. 6. Tension and deformation of the tube with square cross section. 


\subsection{Bending radius}

Four types of tubes were used to perform this analysis with a length of $300 \mathrm{~mm}$ and a total width of $100 \mathrm{~mm}$. What differs in these four tubes is the bending radius of the tubes, thus identifying the following radius sizes: R4, R6, R8 and R10. Their modeling was done in the surface model of the CREO program, to then be given a thickness of $2 \mathrm{~mm}$ directly from the Abaqus simulation program.

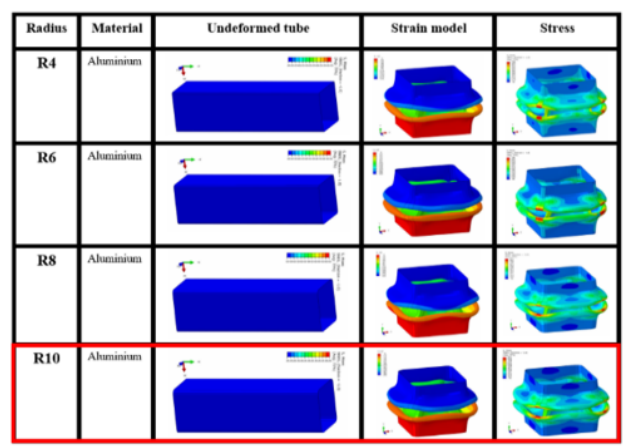

Fig 7. Simulation obtained for bending radius.

For this analysis we started from the best result obtained in the previous analysis. It can be seen from the graph that if we increase the bending radius more and more, the result is more favorable. The $10 \mathrm{~mm}$ bending radius gave the best result, namely $49,500 \mathrm{~mJ}$, compared to the analyzed $6 \mathrm{~mm}$ radius where was obtained a result of only $35,000 \mathrm{~mJ}$. It can also be seen from the graph that the $10 \mathrm{~mm}$ radius continues its absorption even after $200 \mathrm{~mm}$ displacement, while the others stop at a maximum deformation of $195 \mathrm{~mm}$.

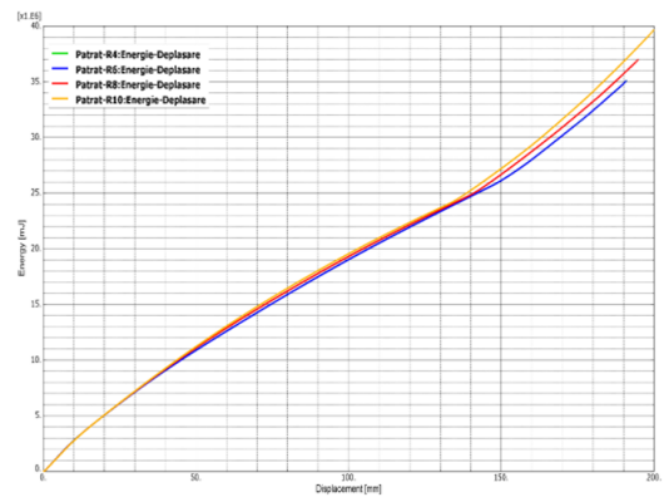

Fig. 8. Simulation graph by bending radius.

The maximum stress at the thin-walled tube with a square cross section and a bending radius of $10 \mathrm{~mm}$ is $871 \mathrm{MPa}$. There is an increase in stresses towards dissociation from the tube without bending radii, but unlike that the flow of the material during deformation is smoother. Its deformation on the $\mathrm{Y}$ axis reaches a value of $200 \mathrm{~mm}$ at the end of the axial compression stress. 


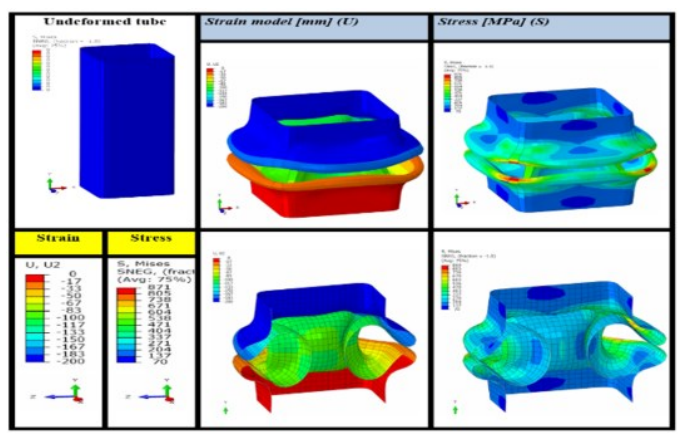

Fig. 9. Tension and deformation of the tube with a bending radius of $10 \mathrm{~mm}$.

\subsubsection{Bending angle}

Three types of tubes were used to perform this analysis, with a length of $300 \mathrm{~mm}$ and a total width of $100 \mathrm{~mm}$. What differs in these three tubes is the bending angle of the tubes, so the following sizes of angles are identified: $93^{\circ}, 95^{\circ}$ and $100^{\circ}$. Their modeling was done in the surface module of the CREO program, to then be given a thickness of $2 \mathrm{~mm}$ directly from the Abaqus simulation program.

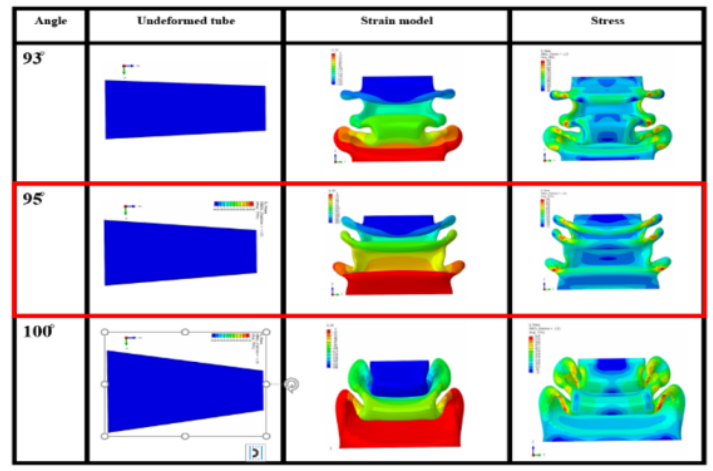

Fig. 10. Simulation obtained for the bending angle.

In this analysis, unlike the first two, bending angles are also applied on the three tubes. The $95^{\circ}$ bending angle offered the best result, namely $37,300 \mathrm{~mJ}$, compared to the analyzed $100^{\circ}$ angle where was obtained a result of $29,500 \mathrm{~mJ}$. It can be seen that a very large angle does not facilitate the absorption of internal energy.

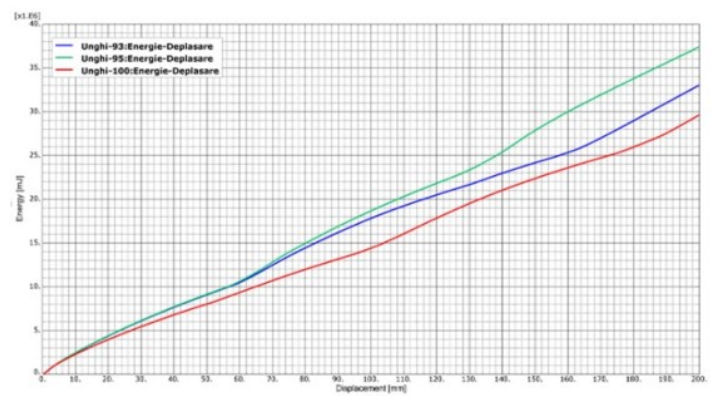

Fig. 11. Simulation graph by bending angle. 
The maximum stress on the thin-walled tube with a square cross section, a bending radius of $10 \mathrm{~mm}$ and a bending angle of $95^{\circ}$ is $980 \mathrm{MPa}$. Its deformation on the $\mathrm{Y}$ axis reaches a value of $200 \mathrm{~mm}$ at the end of the axial compression stress. As can be seen in the Figure 13, the bending angle helps the material to deform and increases its ability to absorb internal energy, the maximum stress being quite high.

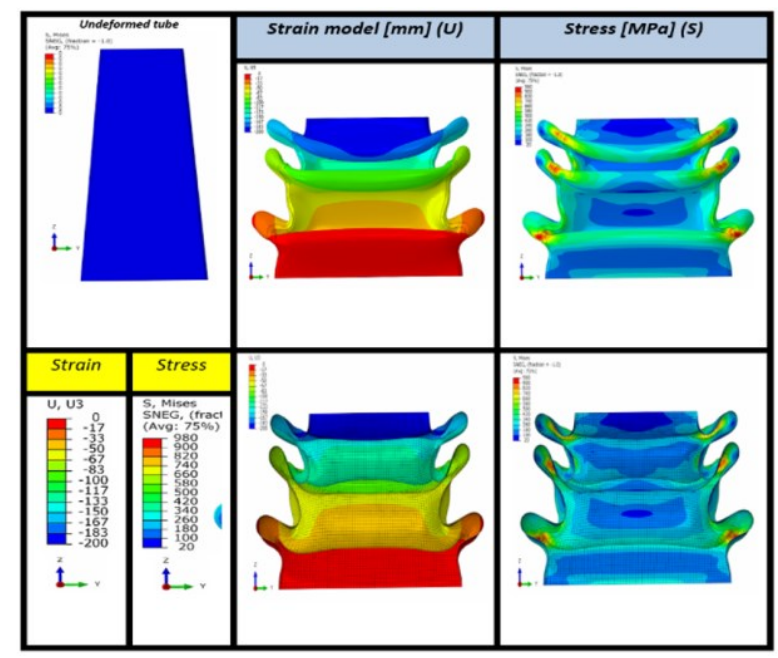

Fig. 12. The tension and the deformation of the tube with a bending angle of $95^{\circ}$.

In order to improve the absorption capacity of the internal energy in case of a frontal impact, these characteristics of the tubes were combined with each other and thus the final constructive form of a thin-walled tube was reached, having the best characteristics resulting from the simulation analyses finite element.

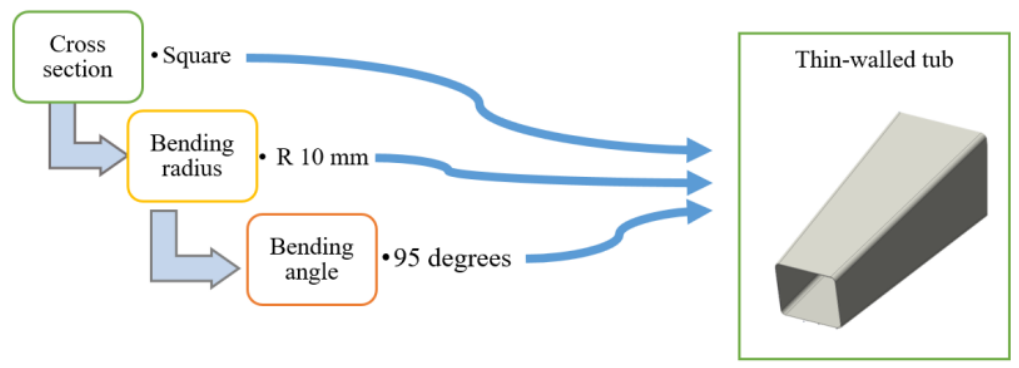

Fig. 13. Elements assigned to the thin-walled tube.

The prototype tube obtained from the analyses is to be combined with structures made by the Origami Engineering technique. It is hoped that this tube together with the Origami core will form a common body capable of reducing the total mass of vehicles, but also by obtaining a compact structure that has very good internal energy absorption properties if subjected to compression. 


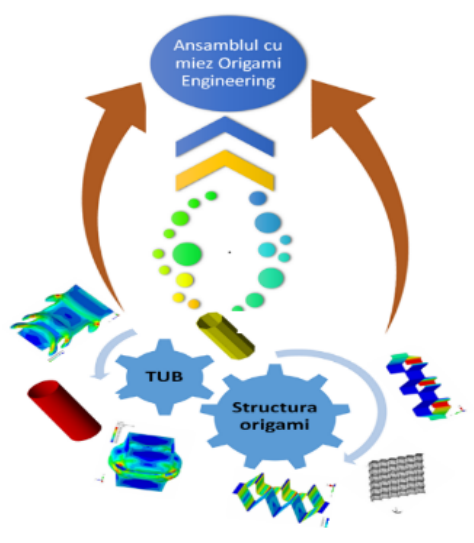

Fig. 14. Origami Engineering core assembly.

\section{Conclusions}

Following the analyses performed with the help of the Abaqus program, three types of characteristics of thin-walled tubes were studied, namely the cross section of the profile, the bending radius and its bending angle. After performing these simulations, it was concluded that the most favorable tube is the one that contains the following characteristics: square cross section, bending radius of $10 \mathrm{~mm}$ and bending angle of 95 degrees. To fulfill the principles of sustainability, try to replace the components that have a high table with light structures made by Origami Engineering technique.

\section{References}

1. I. Rotaru, Acad. J. Manuf. Eng. 12, 95 (2014)

2. D. Dobrota, I. Rotaru, A. Florin, A. Nicolescu, M. Marin, Sustainability 11, 54 (2019)

3. Y. Yu, J. Shi, F. Han, W. Sun, X. Feng, Opt. Express. 28, 37 (2020)

4. K. Yang, S. Xu, S. Zhou, J. Shen, Y.M. Xie, Thin-Walled Struct. 112, 30 (2017)

5. J. Song, Y. Chen, G. Lu, Thin-Walled Struct. 54, 65 (2012)

6. K. Yang, S. Xu, J. Shen, S. Zhou, Y.M. Xie, Thin-Walled Struct. 103, 33 (2016)

7. K.K. Dama, V.S. Babu, R.N. Rao, S. Emmadi, Mater. Today Proc. 4, 28 (2017)

8. Y. Zhang, J. Wang, C. Wang, Y. Zeng, T. Chen, Mater. Des. 158, 14 (2018)

9. C. Reuter, T. Tröster, Thin-Walled Struct. 117, 9 (2017)

10. Q. Shi, X. Shi, J.M. Gattas, S. Kitipornchai, J. Constr. Steel Res. 138, 235 (2017)

11. Z. Gronostajski, P. Kaczyński, S. Polak, B. Bartczak, Thin-Walled Struct. 122, 491 (2018)

12. M.R. Bambach, Compos. Part B Eng. 41, 550 (2010)

13. C. Zhou, S. Ming, C. Xia, B. Wang, X. Bi, P. Hao, M. Ren, Int. J. Mech. Sci. 141, 100 (2018)

14. H. Nikkhah, F. Guo, Y. Chew, J. Bai, J. Song, P. Wang, Thin-Walled Struct. 119, 420 (2017)

15. M.S. Zahran, P. Xue, M.S. Esa, M.M. Abdelwahab, Thin-Walled Struct. 122, 82 (2018)

16. J. Dearden, C. Grames, J. Orr, B.D. Jensen, S.P. Magleby, L.L. Howell, Precis. Eng. 51, 613 (2018)

17. H. Ravi Sankar, V. Parameswaran, Int. J. Impact Eng. 122, 362 (2018)

18. N.S. Ha, G. Lu, X. Xiang, Int. J. Mech. Sci. 148, 421 (2018)

19. G. Sun, X. Tian, J. Fang, F. Xu, G. Li, X. Huang, Int. J. Impact Eng. 78, 137 (2015)

20. E. Mahdi, A.M.S. Hamouda, Mater. Des. 34, 210 (2012)

21. O.M. Qureshi, E. Bertocchi, Z. Qaiser, K. Aslam Awan, Thin-Walled Struct. 75, 7 (2014) 